\title{
Effect of acid functionalized carbon black on mechanical and thermal properties of acrylonitrile-butadiene-styrene/polyaniline blend
}

\author{
Narayan Debnath ${ }^{1}$, Tushar Roy ${ }^{2}$, Mitali Saha ${ }^{1}$ and Kaushik Pal2* \\ ${ }^{1}$ Department of Chemistry, National Institute of Technology Agartala, Tripura -799046, India. \\ ${ }^{2}$ Department of Mechanical and Industrial Engineering, Indian Institute of Technology Roorkee, \\ Roorkee-247667, India. \\ *Email:pl_kshk@yahoo.co.in
}

Polymers and their composites are attracting great attention of the researchers as composites show advanced applications in various fields. The mechanical properties are further enhanced due to loading of carbonaceous materials as fillers in polymer composites. Functionalized fillers show advantages over non-functionalized pure fillers in polymer nanocomposites, so researchers are more interested on modified fillers rather than the unmodified fillers. Carbon black (CB) are being functionalized from last few decades using several methods like oxygen plasma treatment [1], chemical modification by maleic acid and it's derivatives, catecol, resorcinol [2], electro chemical oxidation [3], etc. and heat treatment with mineral acids [2]. CB is one the allotrope of carbon, widely used as filler in polymer nanocomposites, as it is readily available, cheap and easy to prepare. The existence of $\pi$ electron density on the surface of $\mathrm{CB}$ which provides weak van der waal forces, that enables the particles to bind together.

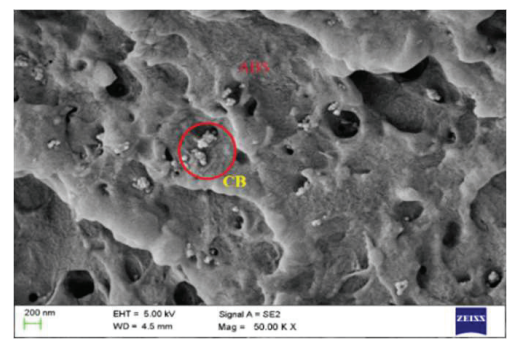

Figure 1: FESEM images of polymer nanocomposite

Mixing of functionalized carbon black (fCB) and combination of fCB-nanoclay (NC) with acrylonitrile butadiene styrene (ABS)/polyaniline (PANi) blend was improved its mechanical and thermal properties. Tensile strength and Young's modulus of the polymer nanocomposites was highly increased. Fourier transformation infrared (FTIR) spectroscopy confirmed the attachment of functional groups on the surface of $\mathrm{CB}$, functionalized by oxidation with mineral acid. Crystallinity and surface morphology of composites were investigated using XRD and field emission scanning electron microscopy
(FESEM). Mechanical properties and thermal properties were analysed through dynamic mechanical analysis (DMA) and universal tensile testing machine and thermo gravimetric analysis (TGA) and differential scanning calorimetry (DSC). Addition of the fillers was decreased the ductility of the polymer nanocomposites considerably but increased thermal stability and melting point.

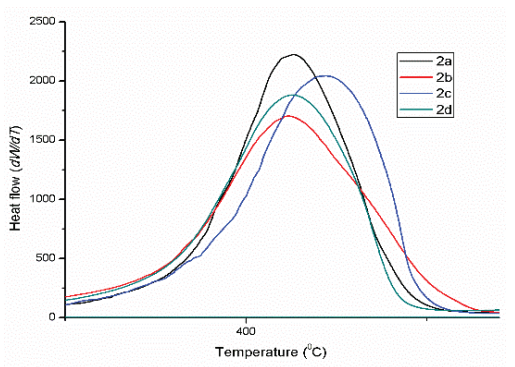

Figure 2: DTG curve of the nanocomposite samples

FESEM images (Figure 1) was taken to see the interfacial adhesion and the extent of mixing among fillers and polymer matrix. The average diameter of fCB particles are about 5-10 nm. FESEM image confirmed good mixing, homogeneous dispersion, uniform distribution and the adhesion of filler particle in polymer matrix. Melting point of the samples was ascertained from the differential thermo gravimetry (DTG) study (Figure 2).

ABS/PANi blend shows good electrical properties but suffers from poor mechanical and thermal properties which make this material less usable. In this paper, authors are interested to increase the mechanical and thermal properties of the said polymer blend.

\section{References}

1. L. E. Cascarini De Torre, E. J. Bottani, A. MartinezAlonso, A. Cuesta, A. B. Garcia, J. M. D. Tascon, Carbon 36 (1998) 277.

2. A. Vijayshankar, K. Pawel, S. Tore, M.Velaug, Front. Mater. Sci. 7 (2013) 302.

3. H. Kiyoshi, N. Yasushi, O. Takao, Carbon 34 (1996) 217. 